\title{
Stability and Hopf Bifurcation in a Delayed Predator-Prey System with Herd Behavior
}

\author{
Chaoqun $\mathrm{Xu}$ and Sanling Yuan \\ College of Science, University of Shanghai for Science and Technology, Shanghai 200093, China \\ Correspondence should be addressed to Sanling Yuan; sanling@usst.edu.cn
}

Received 24 February 2014; Accepted 13 March 2014; Published 8 April 2014

Academic Editor: Yonghui Xia

Copyright (C) 2014 C. Xu and S. Yuan. This is an open access article distributed under the Creative Commons Attribution License, which permits unrestricted use, distribution, and reproduction in any medium, provided the original work is properly cited.

A special predator-prey system is investigated in which the prey population exhibits herd behavior in order to provide a selfdefense against predators, while the predator is intermediate and its population shows individualistic behavior. Considering the fact that there always exists a time delay in the conversion of the biomass of prey to that of predator in this system, we obtain a delayed predator-prey model with square root functional response and quadratic mortality. For this model, we mainly investigate the stability of positive equilibrium and the existence of Hopf bifurcation by choosing the time delay as a bifurcation parameter.

\section{Introduction}

During the last few decades, there has been great interest in the construction and study of models for the population dynamics of predator-prey systems. The classical predatorprey model can be written in the generalized form (see [1])

$$
\begin{gathered}
\frac{d N}{d t}=F(N) N-\phi(N, P) P, \\
\frac{d P}{d t}=[k \phi(N, P)-\mu(P)] P, \\
N(0) \geq 0, \quad P(0) \geq 0,
\end{gathered}
$$

where $N=N(t), P=P(t)$ denote the number or density of the prey and predator species at time $t$, respectively, $F(N)$ is the relative growth function of prey species in the absence of predator, $\mu(P)$ is the relative mortality function of predator species in the absence of prey, $\phi(N, P)$ is the functional response function of the predator to the prey which can be interpreted as the change in the number of prey species attached per unit time per predator as the prey number changes, and $k$ is the biomass conversion or consumption rate.

For system (1), the dynamic behavior is determined by the three crucial components: $F(N), \mu(P)$, and $\phi(N, P)$. Chen et al. in [1] summarized some types of $F(N)$ and $\mu(P)$ which determine the species intrinsic population dynamics (primary production and mortality, resp.). For example,

$$
\begin{gathered}
F(N)=r\left(1-\frac{N}{K}\right), \quad F(N)=r \frac{K-N}{K+\varepsilon N} ; \\
\mu(P) \equiv s, \quad \mu(P)=\frac{a}{b+P} .
\end{gathered}
$$

Functional response function $\phi(N, P)$ also can be classified into many different types: Holling I-IV types $[2,3]$, Beddington-DeAngelis type $[4,5]$, Ivlev type [6], ratiodependent type [7], and the modified forms of these types [818]. Over the years, the mathematical models with different $F(N), \mu(P)$, and $\phi(N, P)$ have been constructed by many researchers for describing different predator-prey systems; see $[2-18]$.

Recently, one kind of predator-prey system with herd behavior is considered by some researchers [19-23]. In this kind of systems, such as herbivores on the large savanna and their large predators, the prey species join together in herds in order to provide a self-defense against predators, and the predator interacts with the prey along the outer corridor of the herd of prey $[20,21]$. Therefore, it is more appropriate to model the response functions of prey in terms of the square root of the number of prey species for this class of systems. In [20], Braza considered a predator-prey system with square root functional response and compared the dynamics of this 
system with the dynamics of predator-prey system that use a typical Lotka-Volterra interaction term.

Moreover, there are some papers concerned with the effects of the form of mortality terms for the dynamics [23-26]. Brentnall et al. [24] pointed out that the mortality term of predator is usually described by "linear form" or "quadratic form" and the quadratic mortality is suited to intermediate predator (such as piscivore). Recently, Yuan et al. [23] studied a spatial predator-prey model with herd behavior and concluded that the Turing pattern is induced by quadratic mortality. Fulton et al. in [25] showed that, in the large and interlinked webs used in ecosystem models, model behavior is far more sensitive to the form of the grazing term than to that of the mortality terms that are close to the modeled food web.

On the other hand, for most of the natural ecosystems, every species does not respond instantaneously to changes in the environment or the interactions with other species within the community. Thus models with delay are much more realistic [27-31], and many researchers paid a lot of attention to the delayed predator-prey systems; see [32-40] and the references cited therein. For example, Xiao and Ruan [34] discussed a delayed predator-prey system with nonmonotonic functional response and showed that there is a B-T singularity for any time delay value and the system can exhibit Hopf bifurcation as the time delay passes through some critical values. $\mathrm{Xu}$ et al. in [36] proposed a delayed periodic L-V type predator-prey system with prey dispersal in two-patch environments and gave the sufficient conditions of the existence, uniqueness, and global stability of positive periodic solution.

Motivated by the above, in the present paper, we are intended to consider a predator-prey system in which the prey species exhibits herd behavior, the predator species is intermediate, and there exists a time delay in the convention of the biomass of prey to that of predator.

This paper is organized as follows. We first introduce our working system in the next section. In Section 3, by analyzing the characteristic equation, we discuss the local stability of a positive equilibrium and study the existence of Hopf bifurcations at the positive equilibrium. In Section 4, using the normal form theory and center manifold argument, the explicit formulae are derived to determine the direction of bifurcations and the properties of bifurcating periodic solutions. Several numerical simulations and a simple discussion are given in the last Section.

\section{The Working System}

Braza [20] proposed the basic predator-prey system with logistic growth in the prey species, linear mortality in the predator species, and a square root functional response function. It is given by

$$
\begin{aligned}
& \frac{d X}{d t}=r X\left(1-\frac{X}{K}\right)-\frac{\alpha \sqrt{X} Y}{1+t_{h} \alpha \sqrt{X}}, \\
& \frac{d Y}{d t}=-s Y+\frac{c \alpha \sqrt{X} Y}{1+t_{h} \alpha \sqrt{X}},
\end{aligned}
$$

where $X=X(t), Y=Y(t)$ denote the number of the prey and predator species at time $t$, respectively. The parameter $r$ is the growth rate of prey species, $K$ is its carrying capacity, $s$ is the death rate of predator species in the absence of prey, $\alpha$ is the search efficiency of predator for prey, $c$ is the biomass conversion rate, and $t_{h}$ is predator's average handling time of prey.

Following References [23] and [24], we choose the quadratic mortality for predator species in system (3). If we further consider the delay fact, system (3) should be modified as the following form:

$$
\begin{aligned}
& \frac{d X}{d t}=r X\left(1-\frac{X}{K}\right)-\frac{\alpha \sqrt{X} Y}{1+t_{h} \alpha \sqrt{X}}, \\
& \frac{d Y}{d t}=-s Y^{2}+\frac{c \alpha \sqrt{X(t-\tau)} Y}{1+t_{h} \alpha \sqrt{X(t-\tau)}},
\end{aligned}
$$

where $-s Y^{2}$ represents the quadratic mortality of predator species and $\tau$ is the time delay which means the growth rate of predator species to depend on the number of the prey species $\tau$ units of time earlier.

By the same way of $[20,23]$, we make some scaling and assume that the average handling time is zero. Then the working system is

$$
\begin{aligned}
& \frac{d x}{d t}=x(1-x)-\sqrt{x} y, \\
& \frac{d y}{d t}=-s y^{2}+c \sqrt{x(t-\tau)} y .
\end{aligned}
$$

The initial conditions for system (5) take the form

$$
\begin{aligned}
& x_{0}(\theta)=\phi(\theta) \geq 0, \quad y_{0}(\theta)=\psi(\theta) \geq 0, \quad \theta \in[-\tau, 0), \\
& x_{0}(0)>0, \quad y_{0}(0)>0 \text {, }
\end{aligned}
$$

where $(\phi(\theta), \psi(\theta)) \in C\left([-\tau, 0], \overline{\mathbb{R}}_{+}^{2}\right), \overline{\mathbb{R}}_{+}^{2}=\left\{\left(r_{1}, r_{2}\right): r_{1} \geq\right.$ $\left.0, r_{2} \geq 0\right\}$.

It is easy to verify that system (5) always has a trivial equilibrium $E_{0}(0,0)$ and a boundary equilibrium $E_{1}(1,0)$ for any feasible parameters. If we assume $c<s$, then system (5) has a unique positive equilibrium $E^{*}\left(x^{*}, y^{*}\right)$, where

$$
x^{*}=1-\frac{c}{s}, \quad y^{*}=\frac{c}{s} \sqrt{x^{*}} .
$$

\section{Stability Analysis and Hopf Bifurcation}

Due to biological interpretation of the system, we only consider the positive equilibrium. In this section, by choosing $\tau$ as the bifurcation parameter and analyzing the corresponding linearized system, we investigate the stability of the positive equilibrium and the effects of the time delay on the dynamics of system (5). 
To study the local stability of the positive equilibrium $E^{*}\left(x^{*}, y^{*}\right)$, we first use the linear transformation $x_{1}=x-x^{*}$, $x_{2}=y-y^{*}$, for which system (5) can be written in the form

$$
\begin{aligned}
& \frac{d x_{1}}{d t}=a_{11} x_{1}+a_{12} x_{2}+\sum_{i+j \geq 2} \frac{f_{i j}^{(1)}}{i ! j !} x_{1}^{i} x_{2}^{j}, \\
& \frac{d x_{2}}{d t}=a_{21} x_{1}(t-\tau)+a_{22} x_{2}+\sum_{i+j \geq 2} \frac{f_{i j}^{(2)}}{i ! j !} x_{1}^{i}(t-\tau) x_{2}^{j},
\end{aligned}
$$

where

$$
\begin{gathered}
a_{11}=\frac{3 c}{2 s}-1, \quad a_{12}=-\sqrt{x^{*}}, \\
a_{21}=\frac{c^{2}}{2 s}, \quad a_{22}=-c \sqrt{x^{*}} \\
f_{i j}^{(1)}=\left.\frac{\partial^{i+j} f^{(1)}\left(x_{1}, x_{2}\right)}{\partial x_{1}^{i} \partial x_{2}^{j}}\right|_{(0,0)}, \quad f_{i j}^{(2)}=\left.\frac{\partial^{i+j} f^{(2)}\left(x_{3}, x_{2}\right)}{\partial x_{3}^{i} \partial x_{2}^{j}}\right|_{(0,0)}, \\
f^{(1)}\left(x_{1}, x_{2}\right)=\left(x_{1}+x^{*}\right)\left(1-x_{1}-x^{*}\right) \\
-\sqrt{x_{1}+x^{*}}\left(x_{2}+y^{*}\right), \\
f^{(2)}\left(x_{3}, x_{2}\right)=-s\left(x_{2}+y^{*}\right)^{2}+c \sqrt{x_{3}+x^{*}}\left(x_{2}+y^{*}\right) .
\end{gathered}
$$

Thus, the positive equilibrium of system (5) is transformed into the zero equilibrium of system (8).

The linear equations corresponding to (8) are given by

$$
\begin{aligned}
& \frac{d x_{1}}{d t}=a_{11} x_{1}+a_{12} x_{2}, \\
& \frac{d x_{2}}{d t}=a_{21} x_{1}(t-\tau)+a_{22} x_{2},
\end{aligned}
$$

and its characteristic equation is

$$
\lambda^{2}-\left(a_{11}+a_{22}\right) \lambda+a_{11} a_{22}-a_{12} a_{21} e^{-\lambda \tau}=0 .
$$

To study the stability of the zero equilibrium and the Hopf bifurcation of system (8), we should analyze the distribution of the roots of characteristic equation (11). A simple calculation shows that $a_{11} a_{22}-a_{12} a_{21}=c\left(x^{*}\right)^{3 / 2}>0$; thus $\lambda=0$ is not a root of (11).

When there is no delay, that is, $\tau=0$, characteristic equation (11) reduces to

$$
\lambda^{2}-\left(a_{11}+a_{22}\right) \lambda+a_{11} a_{22}-a_{12} a_{21}=0 .
$$

If $a_{11}+a_{22}<0$, the two roots of (12) will have always negative real parts. Thus, we can obtain the following result.

Lemma 1. Assume $c<s$ and $a_{11}+a_{22}<0$; then the two roots of characteristic equation (11) with $\tau=0$ have always negative real parts; that is, the zero equilibrium of system (8) with $\tau=0$ is locally asymptotically stable.
Now for $\tau>0$, let $\lambda=i \omega(\omega>0)$ be a root of (11); then $\omega$ satisfies the following equation:

$$
\begin{aligned}
-\omega^{2} & -\left(a_{11}+a_{22}\right) \omega i+a_{11} a_{22}-a_{12} a_{21}(\cos \omega \tau-i \sin \omega \tau) \\
& =0
\end{aligned}
$$

Separating the real and imaginary parts, we have

$$
\begin{aligned}
& -\omega^{2}+a_{11} a_{22}-a_{12} a_{21} \cos \omega \tau=0, \\
& -\left(a_{11}+a_{22}\right) \omega+a_{12} a_{21} \sin \omega \tau=0,
\end{aligned}
$$

which imply that

$$
\omega^{4}+\left(a_{11}^{2}+a_{22}^{2}\right) \omega^{2}+a_{11}^{2} a_{22}^{2}-a_{12}^{2} a_{21}^{2}=0
$$

Notice $a_{11} a_{22}-a_{12} a_{21}>0$; we can find that (15) has no positive roots if $a_{11} a_{22}+a_{12} a_{21} \geq 0$ and has a positive root $\omega_{+}$if $a_{11} a_{22}+$ $a_{12} a_{21}<0$, where

$$
\omega_{+}=\sqrt{\frac{-\left(a_{11}^{2}+a_{22}^{2}\right)+\sqrt{\left(a_{11}^{2}-a_{22}^{2}\right)^{2}+4 a_{12}^{2} a_{21}^{2}}}{2}} .
$$

Defining

$$
\tau_{j}=\frac{1}{\omega_{+}}\left(\arccos \frac{-\left(\omega_{+}\right)^{2}+a_{11} a_{22}}{a_{12} a_{21}}+2 j \pi\right), \quad j=0,1,2, \ldots,
$$

we have the following result.

Lemma 2. Assume $c<s$ and $a_{11}+a_{22}<0$; then the following statements are true.

(1) If $a_{11} a_{22}+a_{12} a_{21} \geq 0$, the roots of (11) have always negative real parts for all $\tau \geq 0$.

(2) If $a_{11} a_{22}+a_{12} a_{21}<0$, (11) has a pair of purely imaginary roots $\pm i \omega_{+}$for $\tau=\tau_{j}$.

Next, under the condition of Lemma 2(2), we will investigate whether the transversality condition is satisfied. Denote by $\lambda(\tau)=\alpha(\tau)+i \omega(\tau)$ the root of (11) near $\tau=\tau_{j}$ satisfying $\alpha\left(\tau_{j}\right)=0, \omega\left(\tau_{j}\right)=\omega_{+}, j=0,1,2, \ldots$ Differentiating the two sides of (11) with respect to $\tau$, we get

$$
2 \lambda \frac{d \lambda}{d \tau}-\left(a_{11}+a_{22}\right) \frac{d \lambda}{d \tau}+a_{12} a_{21} e^{-\lambda \tau}\left(\lambda+\tau \frac{d \lambda}{d \tau}\right)=0
$$

that is

$$
\left(\frac{d \lambda}{d \tau}\right)^{-1}=\frac{2 \lambda-a_{11}-a_{22}}{-a_{12} a_{21} \lambda} e^{\lambda \tau}-\frac{\tau}{\lambda}
$$


We can further get

$$
\begin{aligned}
\left.\operatorname{sign}\left(\frac{d \alpha(\tau)}{d \tau}\right)^{-1}\right|_{\tau=\tau_{j}} \\
=\left.\operatorname{sign} \operatorname{Re}\left(\frac{d \lambda}{d \tau}\right)^{-1}\right|_{\tau=\tau_{j}} \\
=\left.\operatorname{sign} \operatorname{Re}\left(\frac{a_{11}+a_{22}-2 \lambda}{a_{12} a_{21} \lambda} e^{\lambda \tau}-\frac{\tau}{\lambda}\right)\right|_{\tau=\tau_{j}} \\
=\operatorname{sign} \operatorname{Re}\left(\frac{a_{11}+a_{22}-2 i \omega_{+}}{a_{12} a_{21} i \omega_{+}}\left(\cos \omega_{+} \tau_{j}+i \sin \omega_{+} \tau_{j}\right)\right) \\
=\operatorname{sign} \operatorname{Re}\left(\frac{2\left(\omega_{+}^{2}-a_{11} a_{22}\right)}{a_{12}^{2} a_{21}^{2}}+\frac{\left(a_{11}+a_{22}\right)^{2}}{a_{12}^{2} a_{21}^{2}}\right) \\
=\operatorname{sign} \operatorname{Re}\left(\frac{2 \omega_{+}^{2}+a_{11}^{2}+a_{22}^{2}}{a_{12}^{2} a_{21}^{2}}\right)>0 .
\end{aligned}
$$

Then we have the following conclusion.

Lemma 3. Assume $c s, a_{11}+a_{22}<0$, and $a_{11} a_{22}+a_{12} a_{21}<0$; then the transversality conditions are satisfied; that is,

$$
\left.\frac{d \alpha(\tau)}{d \tau}\right|_{\tau=\tau_{j}}>0, \quad j=0,1,2, \ldots
$$

Summarizing the above three lemmas, we can obtain the following theorem on stability and Hopf bifurcation of system (5).

Theorem 4. Assume $c<s$ and $a_{11}+a_{22}<0$. For system (5), the following results are true.

(1) If $a_{11} a_{22}+a_{12} a_{21} \geq 0$, the positive equilibrium $E^{*}$ is locally asymptotically stable for all $\tau \geq 0$.

(2) If $a_{11} a_{22}+a_{12} a_{21}<0$, then $E^{*}$ is locally asymptotically stable when $\tau \in\left[0, \tau_{0}\right)$ and unstable when $\tau>\tau_{0}$ and system (5) undergoes a Hopf bifurcation at $E^{*}$ when $\tau=$ $\tau_{j}, j=0,1,2, \ldots$..

\section{Direction and Stability of the Hopf Bifurcation}

In the previous section, we have obtained the conditions under which a family of periodic solutions bifurcate from the positive equilibrium $E^{*}$ as $\tau$ crosses through the critical values $\tau_{j}$. In this section, we will study the direction of Hopf bifurcation and the stability and period of the bifurcating periodic solutions by applying the normal form theory and the center manifold theorem from Hassard et al. [41]. Since the methods used are standard, we omit the detailed process and only give the main results. The readers can see [41] for more details on the derivation process.
Assume that system (5) undergoes Hopf bifurcations at $E^{*}\left(x^{*}, y^{*}\right)$ for $\tau=\tau_{j}$, and then $\pm i \omega_{+}$are the corresponding purely imaginary roots of characteristic equation (11). Let

$$
\begin{array}{r}
q(\theta)=(1, \alpha)^{T} e^{i \omega_{+} \tau_{j} \theta}, \quad q^{*}(\vartheta)=D\left(1, \alpha^{*}\right) e^{i \omega_{+} \tau_{j} \vartheta} \\
\text { for } \theta, \vartheta \in[-1,0] .
\end{array}
$$

We can choose

$$
\begin{gathered}
\alpha=\frac{i \omega_{+}-a_{11}}{a_{21}}, \quad \alpha^{*}=\frac{-i \omega_{+}-a_{11}}{a_{21}} e^{i \omega_{+}}, \\
D=\frac{1}{1+\alpha^{*} \bar{\alpha}+\tau_{j} \alpha^{*} a_{21} e^{i \omega_{+} \tau_{j}}},
\end{gathered}
$$

such that $q(\theta)$ is the eigenvector of operator $A$ corresponding to $i \omega_{+} \tau_{j}, q^{*}(\vartheta)$ is the eigenvector of operator $A^{*}$ corresponding to $-i \omega_{+} \tau_{j}$, and

$$
\left\langle q^{*}(\vartheta), q(\theta)\right\rangle=1, \quad\left\langle q^{*}(\vartheta), \bar{q}(\theta)\right\rangle=0 .
$$

Thus, we can compute

$$
\begin{gathered}
g_{20}=\bar{D} \tau_{j}\left[\left(f_{20}^{(1)}+2 \alpha f_{11}^{(1)}\right)\right. \\
\left.+\bar{\alpha}^{*}\left(e^{-2 i \omega_{+} \tau_{j}} f_{20}^{(2)}+2 \alpha e^{-i \omega_{+} \tau_{j}} f_{11}^{(2)}+\alpha^{2} f_{02}^{(2)}\right)\right], \\
g_{11}=\bar{D} \tau_{j}\left[\left(f_{20}^{(1)}+(\alpha+\bar{\alpha}) f_{11}^{(1)}\right)\right. \\
\left.+\bar{\alpha}^{*}\left(f_{20}^{(2)}+\left(\alpha e^{i \omega_{+} \tau_{j}}+\bar{\alpha} e^{-i \omega_{+} \tau_{j}}\right) f_{11}^{(2)}+\alpha \bar{\alpha} f_{02}^{(2)}\right)\right], \\
g_{02}=\bar{D} \tau_{j}\left[\left(f_{20}^{(1)}+2 \bar{\alpha} f_{11}^{(1)}\right)\right. \\
\left.\quad+\bar{\alpha}^{*}\left(e^{2 i \omega_{+} \tau_{j}} f_{20}^{(2)}+2 \bar{\alpha} e^{i \omega_{+} \tau_{j}} f_{11}^{(2)}+\bar{\alpha}^{2} f_{02}^{(2)}\right)\right], \\
g_{21}=\bar{D} \tau_{j}\left(w_{1}+\bar{\alpha}^{*} w_{2}\right),
\end{gathered}
$$

where

$$
\begin{aligned}
w_{1}= & \left(2 W_{11}^{(1)}(0)+W_{20}^{(1)}(0)\right) f_{20}^{(1)}+f_{30}^{(1)}+(2 \alpha+\bar{\alpha}) f_{21}^{(1)} \\
& +\left(2 W_{11}^{(2)}(0)+W_{20}^{(2)}(0)+\bar{\alpha} W_{20}^{(1)}(0)+2 \alpha W_{11}^{(1)}(0)\right) f_{11}^{(1)} \\
w_{2}= & \left(2 e^{-i \omega_{+} \tau_{j}} W_{11}^{(1)}(-1)+e^{i \omega_{+} \tau_{j}} W_{20}^{(1)}(-1)\right) f_{20}^{(2)} \\
& +\left(2 \alpha W_{11}^{(2)}(0)+\bar{\alpha} W_{20}^{(2)}(0)\right) f_{02}^{(2)} \\
& +\left(2 \alpha W_{11}^{(1)}(-1)+\bar{\alpha} W_{20}^{(1)}(-1)+e^{i \omega_{+} \tau_{j}} W_{20}^{(2)}(0)\right. \\
& \left.\quad+2 e^{-i \omega_{+} \tau_{j}} W_{11}^{(2)}(0)\right) f_{11}^{(2)} \\
& +\left(2 \alpha+\bar{\alpha} e^{-2 i \omega_{+} \tau_{j}}\right) f_{21}^{(2)}+f_{30}^{(2)} e^{-i \omega_{+} \tau_{j}}
\end{aligned}
$$




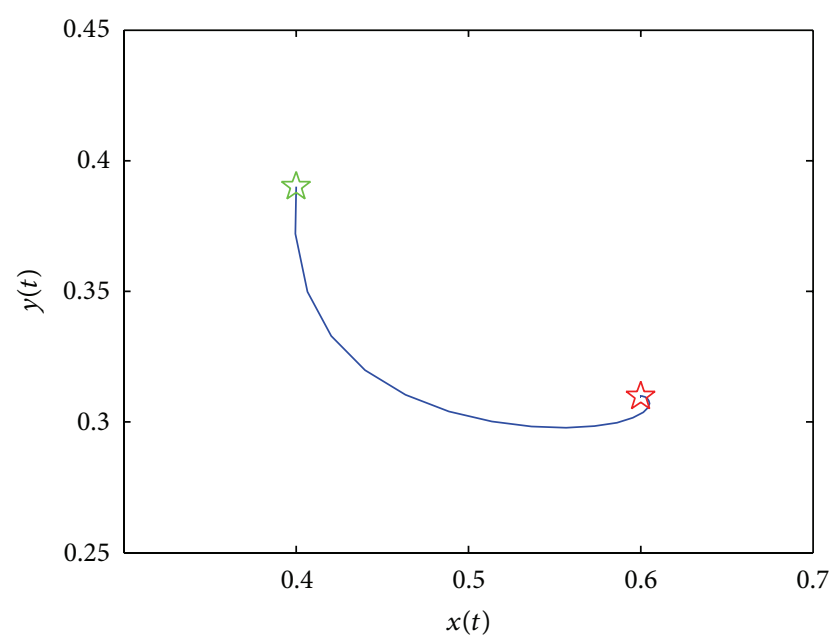

(a)

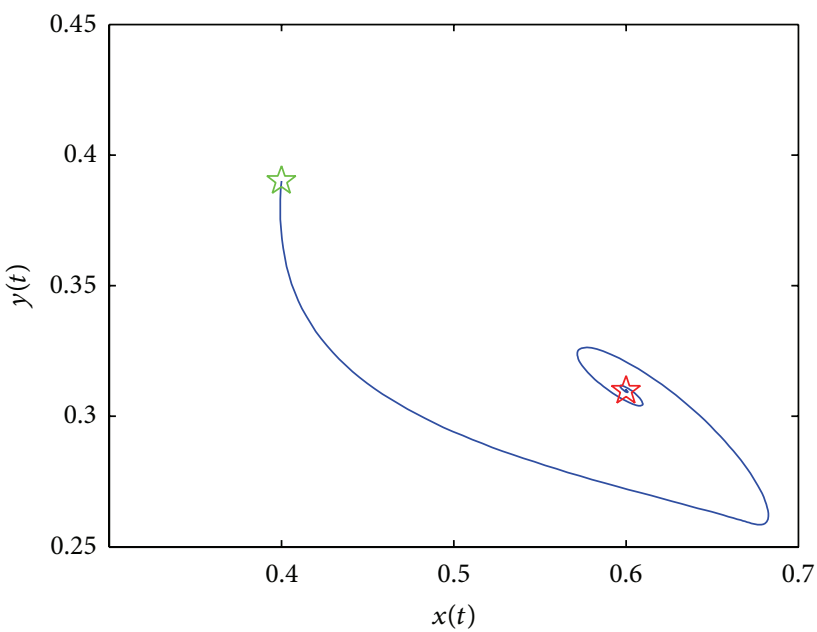

(c)

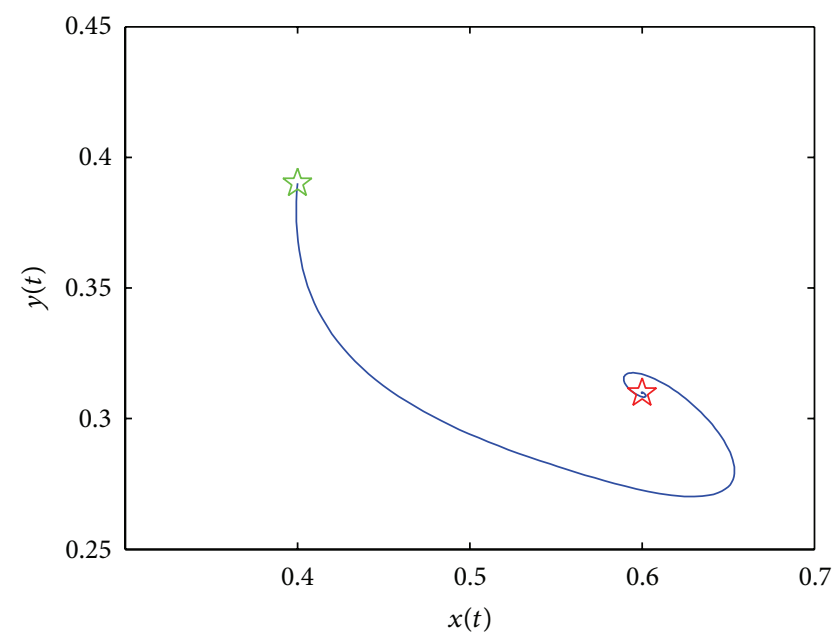

(b)

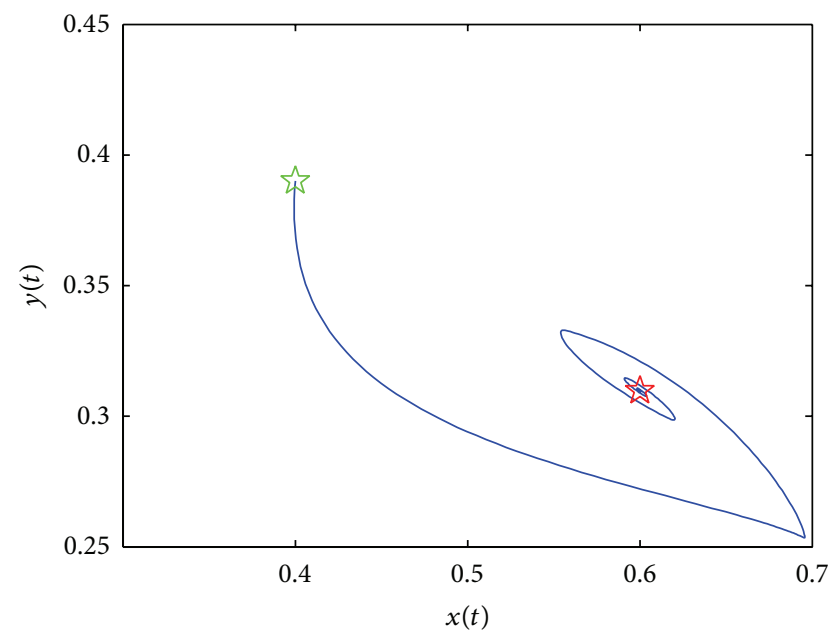

(d)

Figure 1: The phase portraits of system (5). Initial values $x_{0}=0.4, y_{0}=0.39$. Parameters $s=1, c=0.4$. Delay values (a) $\tau=0$, (b) $\tau=5$, (c) $\tau=10$, and (d) $\tau=15$.

$$
\begin{aligned}
W_{20}(\theta) & =\left(\begin{array}{c}
W_{20}^{(1)}(\theta) \\
W_{20}^{(2)}(\theta)
\end{array}\right) \\
& =\frac{i g_{20}}{\omega_{+} \tau_{j}} q(0) e^{i \omega_{+} \tau_{j} \theta}+\frac{i \bar{g}_{20}}{3 \omega_{+} \tau_{j}} \bar{q}(0) e^{-i \omega_{+} \tau_{j} \theta}+E_{1} e^{2 i \omega_{+} \tau_{j} \theta}, \\
W_{11}(\theta) & =\left(\begin{array}{l}
W_{11}^{(1)}(\theta) \\
W_{11}^{(2)}(\theta)
\end{array}\right) \\
& =-\frac{i g_{11}}{\omega_{+} \tau_{j}} q(0) e^{i \omega_{+} \tau_{j} \theta}+\frac{i \bar{g}_{11}}{\omega_{+} \tau_{j}} \bar{q}(0) e^{-i \omega_{+} \tau_{j} \theta}+E_{2}, \\
E_{1}= & \left(\begin{array}{ll}
2 i \omega_{+}-a_{11} & -1 \\
-a_{21} e^{-2 i \omega_{+}} & 2 i \omega_{+}-a_{22}
\end{array}\right) \\
\times & \left(\begin{array}{cc}
-1) \\
e^{-2 i \omega_{+} \tau_{j}} f_{20}^{(2)}+2 \alpha e^{-i \omega_{+} \tau_{j}} f_{11}^{(2)}+\alpha^{2} f_{02}^{(2)}
\end{array}\right),
\end{aligned}
$$$$
E_{2}=-\left(\begin{array}{ll}
a_{11} & a_{12} \\
a_{21} & a_{22}
\end{array}\right)^{-1}
$$$$
\times\left(\begin{array}{c}
f_{20}^{(1)}+(\alpha+\bar{\alpha}) f_{11}^{(1)} \\
f_{20}^{(2)}+\left(\alpha e^{i \omega_{+} \tau_{j}}+\bar{\alpha} e^{-i \omega_{+} \tau_{j}}\right) f_{11}^{(2)}+\alpha \bar{\alpha} f_{02}^{(2)}
\end{array}\right) .
$$

Now, let

$$
c_{1}(0)=\frac{i}{2 \omega_{+} \tau_{j}}\left(g_{20} g_{11}-2\left|g_{11}\right|^{2}-\frac{\left|g_{02}\right|^{2}}{3}\right)+\frac{g_{21}}{2},
$$

which can be determined by the parameters in system (5). Defining

$$
\mu_{2}=-\frac{\operatorname{Re}\left\{c_{1}(0)\right\}}{\operatorname{Re}\left\{\lambda^{\prime}\left(\tau_{j}\right)\right\}},
$$



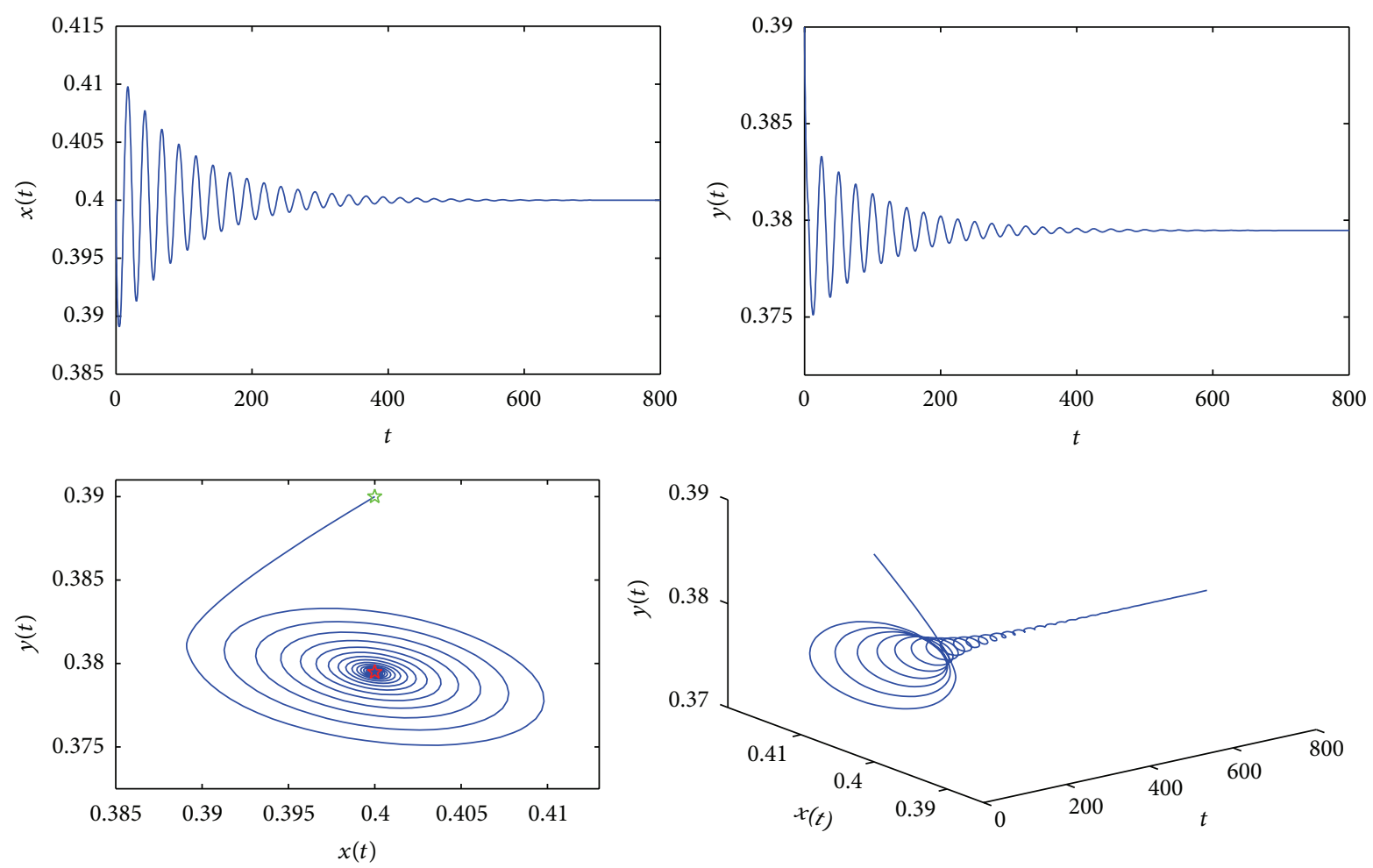

FIGURE 2: The trajectories and phase portrait of system (5). Initial values $x_{0}=0.4, y_{0}=0.39$. Parameters $s=1, c=0.6$. Delay value $\tau=5.248$.

$$
\begin{aligned}
& \beta_{2}=2 \operatorname{Re}\left\{c_{1}(0)\right\}, \\
& T_{2}=-\frac{\operatorname{Im}\left\{c_{1}(0)\right\}+\mu_{2} \operatorname{Im}\left\{\lambda^{\prime}\left(\tau_{j}\right)\right\}}{\omega_{+} \tau_{j}},
\end{aligned}
$$

we have the following result.

Theorem 5. The direction of the Hopf bifurcation is determined by $\mu_{2}$ : if $\mu_{2}>0\left(\mu_{2}<0\right)$, then the Hopf bifurcation is supercritical (subcritical) and the bifurcating periodic solutions exist for $\tau>\tau_{0}\left(\tau<\tau_{0}\right) ; \beta_{2}$ determines the stability of the bifurcating periodic solutions: the bifurcating periodic solutions are stable (unstable) if $\beta_{2}<0\left(\beta_{2}>0\right)$; and $T_{2}$ determines the period of the bifurcating periodic solutions: the period increases (decreases) if $T_{2}>0\left(T_{2}<0\right)$.

\section{Numerical Simulations and Discussions}

In this section, we present some numerical simulations to verify our theoretical results proved in previous sections by using MATLAB DDE solver. We simulate the system (5) with the initial value $\left(x_{0}, y_{0}\right)=(0.4,0.39)$. In Figures $1-3$ the initial point $\left(x_{0}, y_{0}\right)$ and the positive equilibrium $E^{*}$ are represented by green star and red star, respectively.

First, we choose parameters $s=1, c=0.4$. In this case, system (5) has only one positive equilibrium $E^{*}=$ $(0.6,0.3098)$. Simple calculations show that $a_{11}+a_{22}=$ $-0.7098<0, a_{11} a_{22}+a_{12} a_{21}=0.062>0$, which satisfy the conditions of Theorem 4(1). By Theorem 4(1), we expect that $E^{*}$ is stable for any delay value. We further choose four different delay values: $\tau_{a}=0, \tau_{b}=5, \tau_{c}=10$, and $\tau_{d}=$ 15. The computation simulations are depicted in Figure 1. Obviously, the computation simulations can support the result of Theorem 4(1).

Next, we choose parameters $s=1, c=0.6$. The positive equilibrium of system (5) is $E^{*}=(0.4,0.3795)$. It is easy to show that $a_{11}+a_{22}=-0.4795<0, a_{11} a_{22}+a_{12} a_{21}=-0.0759<$ 0 , which satisfy the conditions of Theorem 4(2). From the formulae in the previous section, we can compute the values of $\tau_{0}, \mu_{2}, \beta_{2}$, and $T_{2}$ as

$$
\begin{aligned}
& \tau_{0}=6.048, \quad \mu_{2}=130888, \\
& \beta_{2}=-503.3349, \quad T_{2}=681.8543,
\end{aligned}
$$

from which we conclude that $E^{*}$ is asymptotically stable for $\tau<\tau_{0}$ and the Hopf bifurcation of system (5) occurring at the critical value $\tau_{0}$ is supercritical and the bifurcating periodic solution exists when $\tau$ crosses $\tau_{0}$ to the right; also the bifurcating periodic solution is stable. By taking $\tau=$ $5.248<\tau_{0}$ and $\tau=6.049>\tau_{0}$, we can show the computation simulations in Figures 2 and 3, respectively. It is easy to see that the computation simulations support the results of Theorem 4(2) and Theorem 5.

In the present paper, we have considered a delayed predator-prey system in which the prey species exhibits herd behavior and the predator species with quadratic mortality. Our research shows that, for system (5), the positive equilibrium $E^{*}$ is always asymptotically stable under certain 

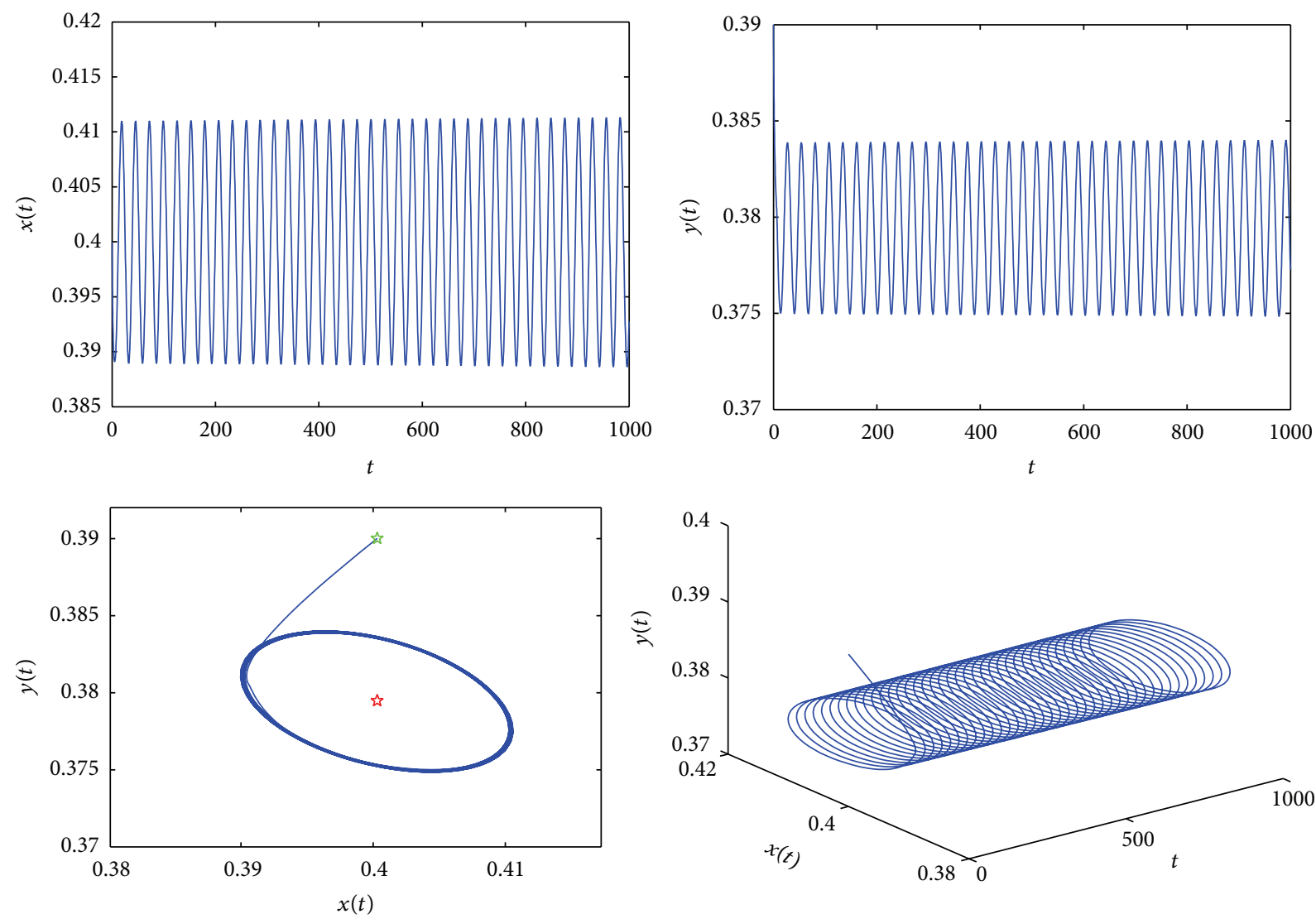

FIgURE 3: The trajectories and phase portrait of system (5). Initial values $x_{0}=0.4, y_{0}=0.39$. Parameters $s=1, c=0.6$. Delay value $\tau=6.049$.

conditions (see Theorem 4(1)). In other words, under such conditions, the dynamics behavior of the corresponding ODE system is robust with respect to the time delay. But, under some other conditions (see Theorem 4(2)), $E^{*}$ will lose its stability and Hopf bifurcations occur when the delay $\tau$ passes through some critical values. In this case, the time delay has an important effect for the system dynamics.

Our results may enrich the dynamics in the predatorprey system and help us to better understand the interaction of predator with prey in a real ecosystem. Further studies are necessary to analyze the dynamics of more realistic but complex systems, such as delayed diffusive predator-prey system with herd behavior.

\section{Conflict of Interests}

The authors declare that there is no conflict of interests regarding the publication of this paper.

\section{Acknowledgments}

The authors wish to thank the handling editor and reviewers for their valuable comments and suggestions which lead to truly significant improvement of the paper. This research is supported by the National Natural Science Foundation of China (no. 11271260), Shanghai Leading Academic Discipline Project (no. XTKX2012), Innovation Program of Shanghai
Municipal Education Commission (no. 13ZZ116), and the Innovation Fund Project For Graduate Student of Shanghai (no. JWCXSL1401).

\section{References}

[1] L. Chen, X. Song, and Z. Lu, Mathematical Ecological Models and Research Methods, Sichuan Science and Technology Press, Chengdu, China, 2003.

[2] C. S. Holling, "The functional response of predators to prey density and its role in mimicry and population regulation," Memoirs of the Entomological Society of Canada, vol. 45, supplement S45, pp. 5-60, 1965.

[3] J. F. Andrews, "A mathematical model for the continuous culture of microorganisms utilizing inhibitory substrates," Biotechnology and Bioengineering, vol. 10, no. 6, pp. 707-723, 1968.

[4] J. Beddington, "Mutual interference between parasites or predators and its effect on searching effciency," Journal of Animal Ecology, vol. 44, no. 1, pp. 331-340, 1975.

[5] D. L. DeAngelis, R. A. Goldsten, and R. V. O’Neill, "A model for trophic interaction,” Ecology, vol. 56, no. 4, pp. 881-892, 1975.

[6] R. E. Kooij and A. Zegeling, "A predator-prey model with Ivlev's functional response," Journal of Mathematical Analysis and Applications, vol. 198, no. 2, pp. 473-489, 1996.

[7] R. Arditi and L. R. Ginzburg, "Coupling in predator-prey dynamics: ratio-dependence," Journal of Theoretical Biology, vol. 139, no. 3, pp. 311-326, 1989. 
[8] Y. Kuang and E. Beretta, "Global qualitative analysis of a ratiodependent predator-prey system," Journal of Mathematical Biology, vol. 36, no. 4, pp. 389-406, 1998.

[9] S. Ruan and D. Xiao, "Global analysis in a predator-prey system with nonmonotonic functional response," SIAM Journal on Applied Mathematics, vol. 61, no. 4, pp. 1445-1472, 2000.

[10] D. Xiao and H. Zhu, "Multiple focus and Hopf bifurcations in a predator-prey system with nonmonotonic functional response," SIAM Journal on Applied Mathematics, vol. 66, no. 3, pp. 802819, 2006.

[11] H. Zhu, S. A. Campbell, and G. S. K. Wolkowicz, "Bifurcation analysis of a predator-prey system with nonmonotonic functional response," SIAM Journal on Applied Mathematics, vol. 63, no. 2, pp. 636-682, 2002.

[12] X.-A. Zhang, L. Chen, and A. U. Neumann, "The stagestructured predator-prey model and optimal harvesting policy," Mathematical Biosciences, vol. 168, no. 2, pp. 201-210, 2000.

[13] M. A. Aziz-Alaoui and M. D. Okiye, "Boundedness and global stability for a predator-prey model with modified Leslie-Gower and Holling-type II schemes," Applied Mathematics Letters, vol. 16, no. 7, pp. 1069-1075, 2003.

[14] A. F. Nindjin, M. A. Aziz-Alaoui, and M. Cadivel, "Analysis of a predator-prey model with modified Leslie-Gower and Hollingtype II schemes with time delay," Nonlinear Analysis: Real World Applications, vol. 7, no. 5, pp. 1104-1118, 2006.

[15] L. Han, Z. Ma, and H. W. Hethcote, "Four predator prey models with infectious diseases," Mathematical and Computer Modelling, vol. 34, no. 7-8, pp. 849-858, 2001.

[16] R. Xu and Z. Ma, "Stability and Hopf bifurcation in a predatorprey model with stage structure for the predator," Nonlinear Analysis: Real World Applications, vol. 9, no. 4, pp. 1444-1460, 2008.

[17] M. Liu and K. Wang, "Global stability of stage-structured predator-prey models with Beddington-DeAngelis functional response," Communications in Nonlinear Science and Numerical Simulation, vol. 16, no. 9, pp. 3792-3797, 2011.

[18] C. Ji, D. Jiang, and X. Li, "Qualitative analysis of a stochastic ratio-dependent predatorprey system," Journal of Computational and Applied Mathematics, vol. 235, no. 5, pp. 1326-1341, 2011.

[19] V. Ajraldi, M. Pittavino, and E. Venturino, "Modeling herd behavior in population systems," Nonlinear Analysis: Real World Applications, vol. 12, no. 4, pp. 2319-2338, 2011.

[20] P. A. Braza, "Predator-prey dynamics with square root functional responses," Nonlinear Analysis: Real World Applications, vol. 13, no. 4, pp. 1837-1843, 2012.

[21] E. Venturino and S. Petrovskii, "Spatiotemporal behavior of a prey-predator system with a group defense for prey," Ecological Complexity, vol. 14, pp. 37-47, 2013.

[22] S. Belvisi and E. Venturino, "An ecoepidemic model with diseased predators and prey group defense," Simulation Modelling Practice and Theory, vol. 34, pp. 144-155, 2013.

[23] S. Yuan, C. Xu, and T. Zhang, "Spatial dynamics in a predatorprey model with herd behavior," Chaos, vol. 23, no. 3, Article ID 033102, 2013.

[24] S. J. Brentnall, K. J. Richards, J. Brindley, and E. Murphy, "Plankton patchiness and its effect on larger-scale productivity," Journal of Plankton Research, vol. 25, no. 2, pp. 121-140, 2003.

[25] E. A. Fulton, A. D. M. Smith, and C. R. Johnson, "Mortality and predation in ecosystem models: is it important how these are expressed?" Ecological Modelling, vol. 169, no. 1, pp. 157-178, 2003.
[26] M. Baurmann, T. Gross, and U. Feudel, "Instabilities in spatially extended predator-prey systems: spatio-temporal patterns in the neighborhood of Turing-Hopf bifurcations," Journal of Theoretical Biology, vol. 245, no. 2, pp. 220-229, 2007.

[27] N. MacDonald, Biological Delay Systems: Linear Stability Theory, Cambridge University Press, Cambridge, UK, 1989.

[28] J. K. Hale and S. M. V. Lunel, Introduction to FunctionalDifferential Equations, Springer, New York, NY, USA, 1993.

[29] Y. Kuang, Delay Differential Equations with Applications in Population Dynamics, Academic Press, Boston, Mass, USA, 1993.

[30] J. Wu, Theory and Applications of Partial Functional-Differential Equations, Springer, New York, NY, USA, 1996.

[31] R. Xu, Q. Gan, and Z. Ma, "Stability and bifurcation analysis on a ratio-dependent predator-prey model with time delay," Journal of Computational and Applied Mathematics, vol. 230, no. 1, pp. 187-203, 2009.

[32] Y. Song and S. Yuan, "Bifurcation analysis in a predatorprey system with time delay," Nonlinear Analysis: Real World Applications, vol. 7, no. 2, pp. 265-284, 2006.

[33] Y. Song and J. Wei, "Local Hopf bifurcation and global periodic solutions in a delayed predator-prey system," Journal of Mathematical Analysis and Applications, vol. 301, no. 1, pp. 1-21, 2005.

[34] D. Xiao and S. Ruan, "Multiple bifurcations in a delayed predator-prey system with nonmonotonic functional response," Journal of Differential Equations, vol. 176, no. 2, pp. 494-510, 2001.

[35] S. Ruan, "Absolute stability, conditional stability and bifurcation in Kolmogorov-type predator-prey systems with discrete delays," Quarterly of Applied Mathematics, vol. 59, no. 1, pp. 159173, 2001.

[36] R. Xu, M. A. J. Chaplain, and F. A. Davidson, "Periodic solutions for a delayed predator-prey model of prey dispersal in twopatch environments," Nonlinear Analysis: Real World Applications, vol. 5, no. 1, pp. 183-206, 2004.

[37] Z. Liu and R. Yuan, "Stability and bifurcation in a delayed predator-prey system with Beddington-DeAngelis functional response," Journal of Mathematical Analysis and Applications, vol. 296, no. 2, pp. 521-537, 2004.

[38] M. Fan and K. Wang, "Periodicity in a delayed ratio-dependent predator-prey system," Journal of Mathematical Analysis and Applications, vol. 262, no. 1, pp. 179-190, 2001.

[39] E. Beretta and Y. Kuang, "Global analyses in some delayed ratiodependent predator-prey systems," Nonlinear Analysis: Theory, Methods and Applications, vol. 32, no. 3, pp. 381-408, 1998.

[40] T. Zhao, Y. Kuang, and H. L. Smith, "Global existence of periodic solutions in a class of delayed gause-type predator-prey systems," Nonlinear Analysis: Theory, Methods and Applications, vol. 28, no. 8, pp. 1373-1394, 1997.

[41] B. D. Hassard, N. D. Kazarinoff, and Y. H. Wan, Theory and Applications of Hopf Bifurcation, Cambridge University Press, Cambridge, UK, 1981. 


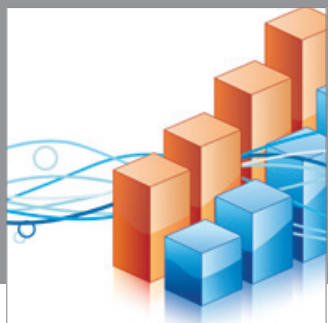

Advances in

Operations Research

mansans

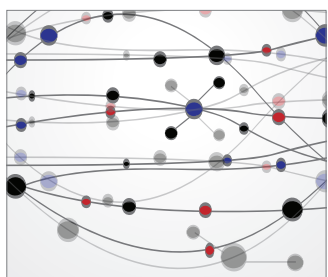

The Scientific World Journal
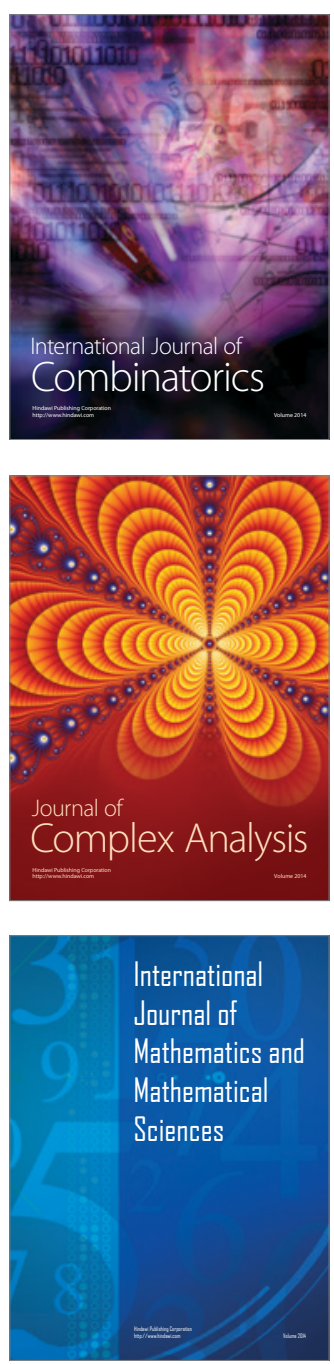
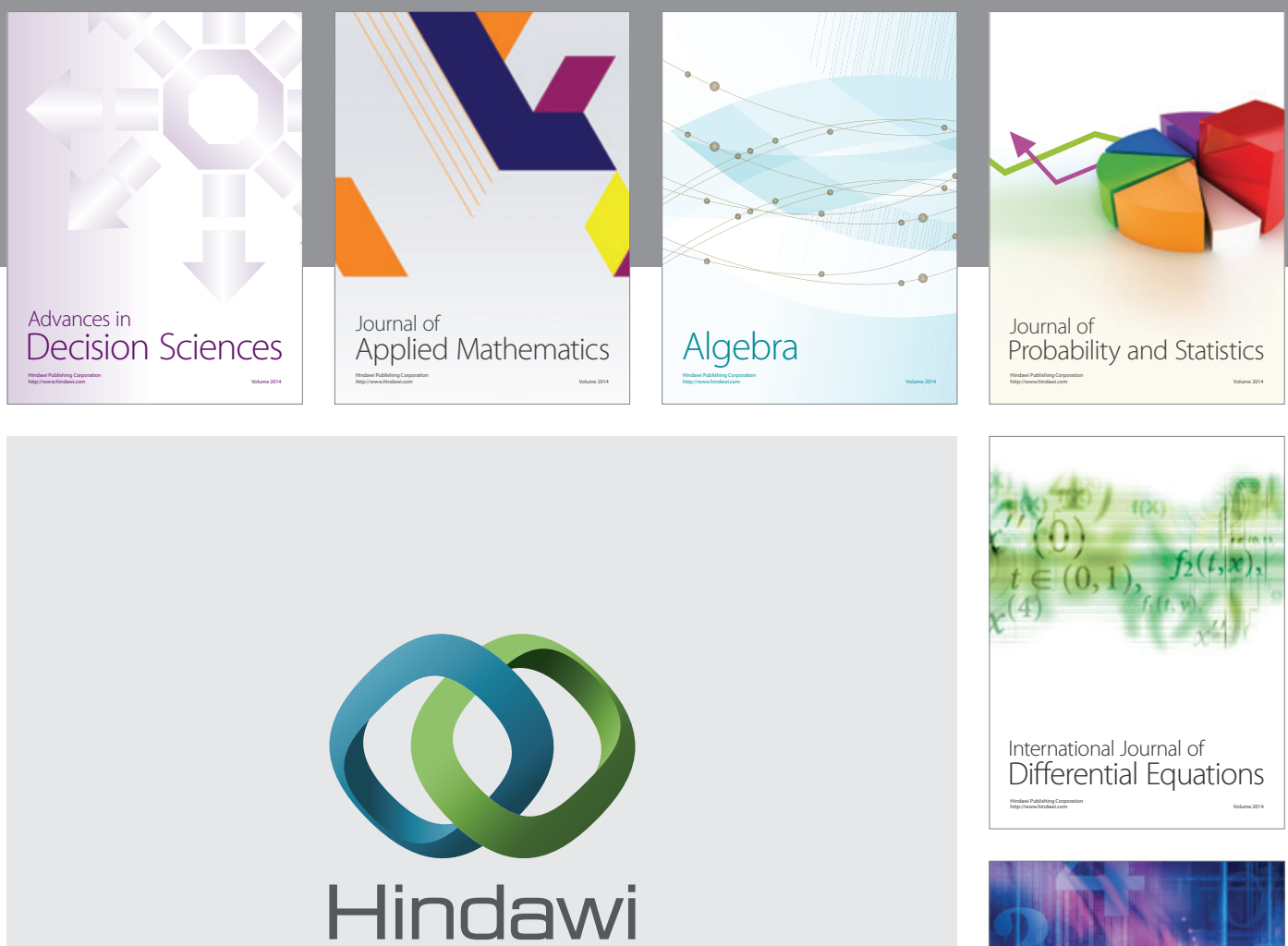

Submit your manuscripts at http://www.hindawi.com
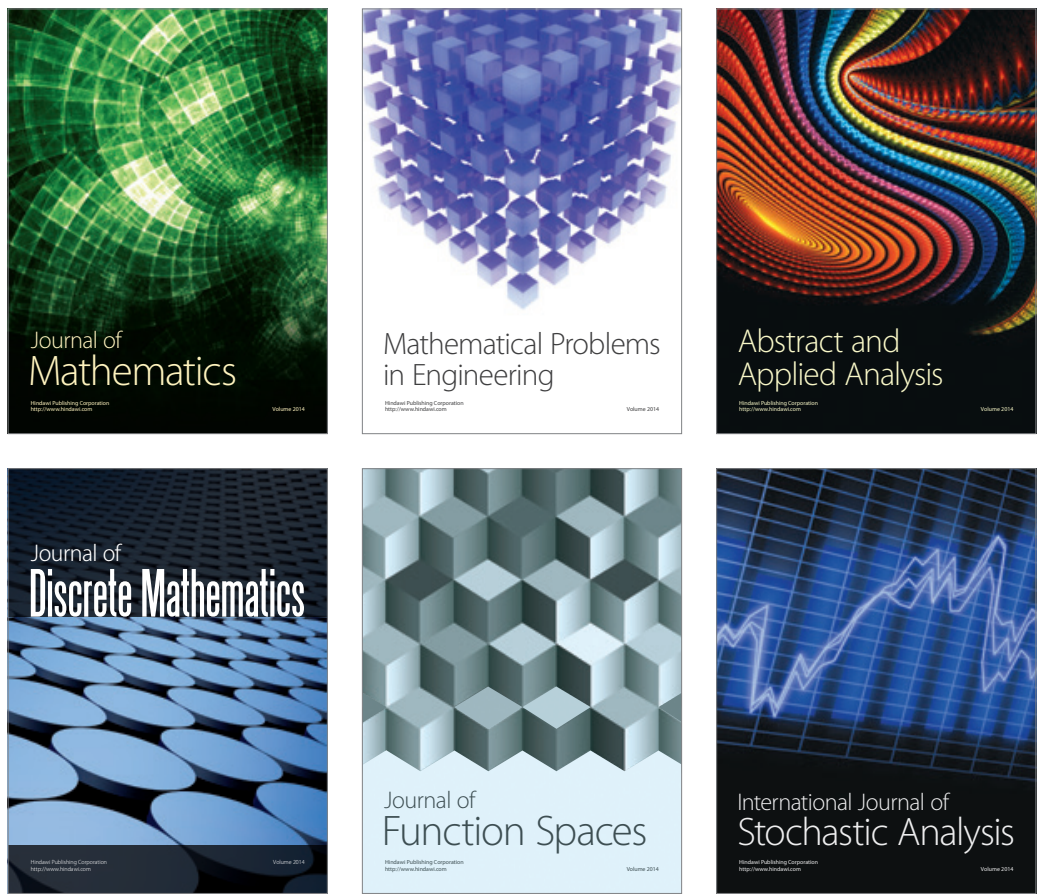

Journal of

Function Spaces

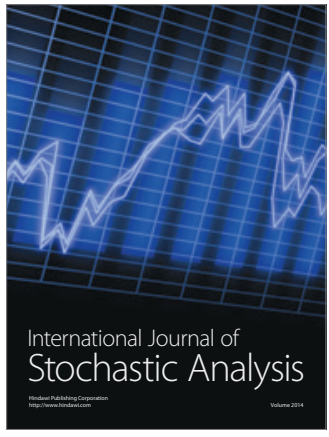

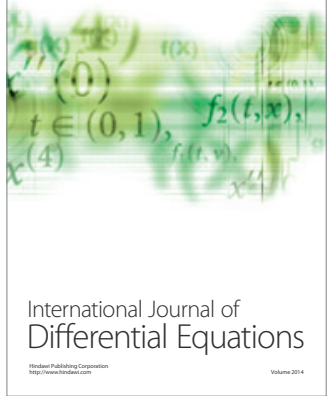
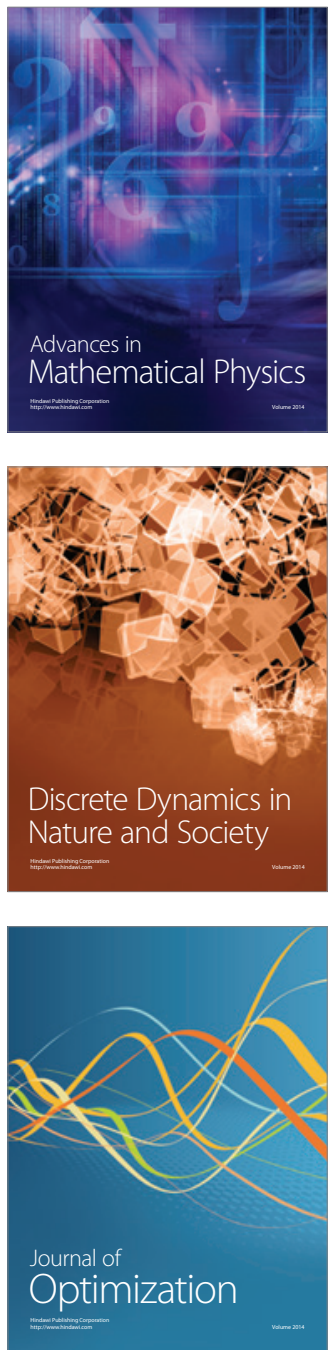